\title{
Pre-congress
}

Juntendo Medical Journal

2010. 56 (3), 251 256

\section{Sportology and High Intensity Interval Walking Training in Aging Society}

\author{
Hiroshi NOSE*1) 2)
}

Toshiaki YAMAZAKI*1)3)

\author{
Mayuko MORIKAWA*1) 2)
}

KEN-ICHI NEMOTO*1) 4)

\author{
ShizUe MASUKI*1)
}

KaZunobu OKAZAKI*1) 5)

\author{
Yoshi-IChiro KAMIJO*1) HirokazU GEN-NO*2) 3)
}

\begin{abstract}
Faced with social problems such as global warming and rapidly aging society, the solutions have been expected in "sportology". Humans became widely distributed on the earth from their birth by acquiring abilities to walk in an upright position and to adapt themselves to various natural environments. However, seeking a 'comfortable environment' in modern civilization has deteriorated these genetic characteristics of humans, and the consumption of resources and energy to acquire such a 'comfortable environment' has induced global warming-associated natural disasters and the destruction of social order. To halt this vicious cycle, we may reactivate the genetic characteristics in humans by doing sports.

To assess this, we have developed a health promotion program for middle aged and elder people, Jukunen Taiikudaiiku Program, in cooperation with the Japanese government, developed high-intensity interval walking training (IWT), and examined the physical and mental effects on 4,000 people for these 5 years. We found that IWT for 4 months increased physical fitness by 10$20 \%$, decreased the indices of life-style related diseases by $10-20 \%$. Since a prescription of IWT can be conducted by using an IT network system called e-Health Promotion System, the participants in the program were able to receive the prescription even if they lived remote from trainers, enabling them to perform IWT at their favored places and times, and also at low cost. Moreover, we found some single nucleotide polymorphisms closely related to inter-individual differences in the responses to IWT. Finally, we assessed a cost vs effect relationship of IWT and found that 60,000 JPY investment per year for individuals reduced their heath care cost by $120,000 \mathrm{JPY}, 20 \%$ of total health care cost per year for the population.

These results suggest that exercise prescription promote health by reactivating the genes unique for human beings, which is quite matched to the goal of "sportology".
\end{abstract}

\section{Introduction}

* 1) Department of Sports Medical Sciences, Shinshu University Graduate School of Medicine, Nagano, Japan

*2) Jukunen Taiikudaigaku Research Center, Nagano, Japan

* 3) Kissei Comtec Co., Ltd, Nagano, Japan

* 4) Faculty of Human Health, Matsumoto University, Nagano, Japan

* 5) Research Center for Urban Health and Sports, Osaka City University, Osaka, Japan

[Received Apr. 12, 2010]
One of the serious problems in advanced aging society is high health care cost for elderly people. In Japan, the population over 65 years old is estimated to increase from $20.8 \%$ in 2006 to $30.5 \%$ in 2025 (http://www.ipss.go.jp/index-e.html (09/07/ 16)) . Consequently, health care cost increased from 17.1 trillion JPY ( $\sim 171$ billion USD) to 56 trillion JPY ( $\sim 560$ billion USD) in 2025 (http://wwwl. mhlw.go.jp/english/wp_5/vol1/p2c3s2.html (09/07/ 16)) . 


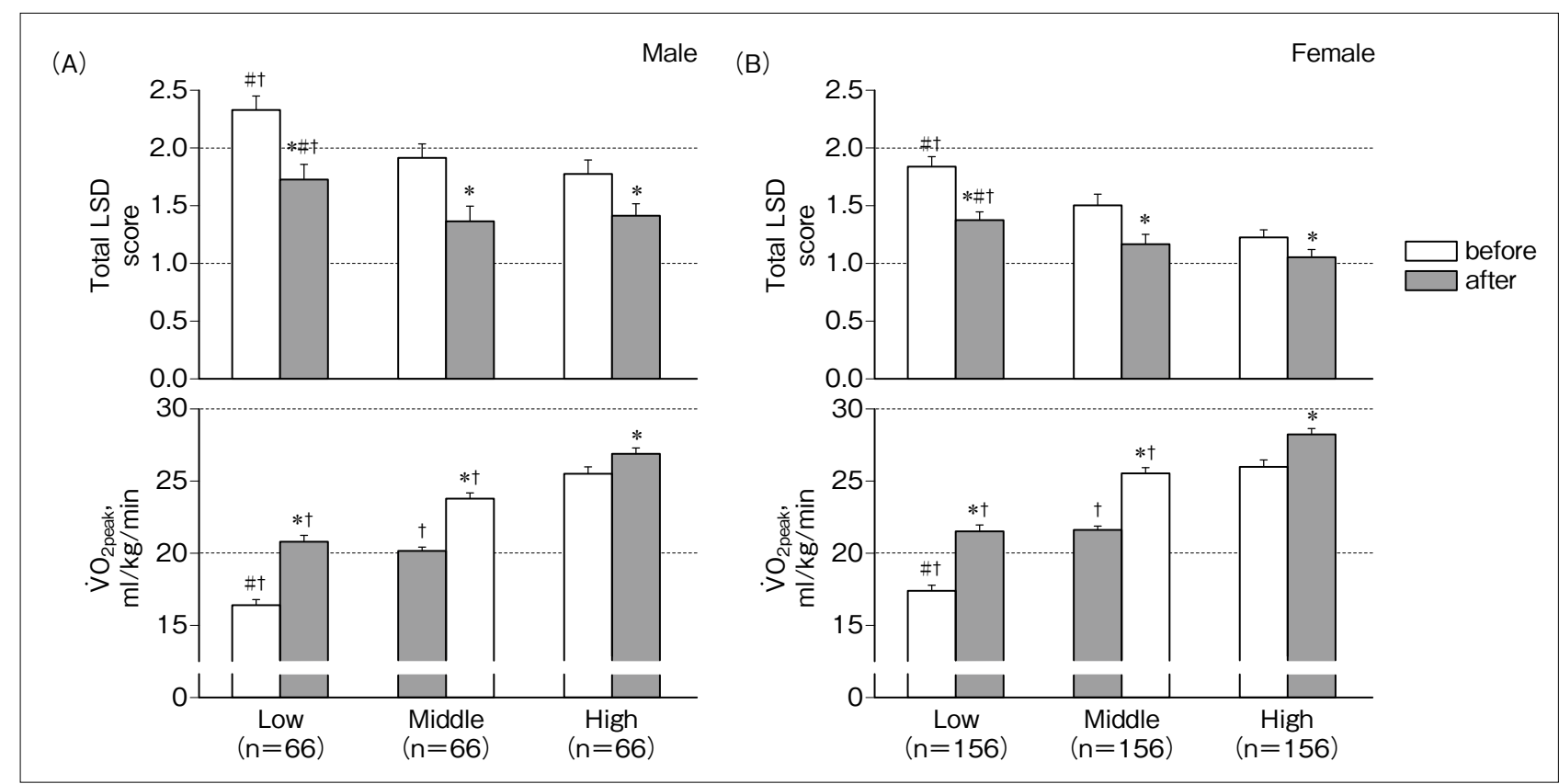

Figure-1 Total lifestyle related disease (LSD) score and peak aerobic capacity for walking $\left(\mathrm{VO}_{2 \text { peak }}\right)$ before and after interval walking training in males $(\mathrm{A})$ and females $(\mathrm{B})$. When the subjects were divided equally into 3 groups according to $\mathrm{WVO}_{2 \text { peak }}$, the score was lower in higher $\mathrm{WVO}_{2 \text { peak }}$ groups. After interval walking training for 4 months, the score decreased as $\mathrm{WVO}_{2 \text { peak }}$ increased in every group. *'s : vs. before training at $\mathrm{p}<$ 0.05, \#'s : vs. Middle group at $\mathrm{p}<0.05,+$ 's : vs. High group at $\mathrm{p}<0.05$. From Morikawa et al., 2009.

To prevent this, the Japanese government has issued the law to reform medial system for treatment to preventive medicine (Health Insurance Bureau, 2007), where people over 40 years old were obligated to receive health check and, if abnormal remarks are indicated, they are encouraged to receive exercise as well as nutritional prescription at a health care office close to their home. However, although exercise prescription should be done based the individual physical fitness (Armstrong et al., 2006) , no guidelines have been provided by the government.

To solve this problem, we started a health promotion program by exercise for people $\geqq 40$ years old named "Jukunen Taiikudaigaku Program" since 1997 and developed 3 techniques for exercise training by middle-aged and older people ; 1) interval walking training (IWT), 2) portable calorie meter and 3) e-Health Promotion System. By using these, we have accumulated the database (DB) on the effects of interval walking training on the indices of age and life-style related diseases (LSD) in more than 4,000 subjects with health care cost in a part of them. Moreover, we have accumulated the DB on DNA in more than 1,400 subjects to exam- ine whether genetic variance caused any inter-individual variation in responses to the training which would be useful to develop an appropriate exercise prescription met with individual genetic characteristics in future.

In this review, we would like to introduce the achievements by the Jukunen Taiikudaigaku Program.

\section{Interval walking training}

Moderately paced walking at 10,000 steps/day, every day has been believed to prevent life-style related diseases in middle-aged and older people. However, the walking may not be intense enough to increase physical fitness including peak aerobic capacity $\left(\mathrm{VO}_{2 \text { peak }}\right)$. Indeed, a higher intensity of aerobic exercise more than $50 \% \mathrm{VO}_{2 \text { peak }}$ has been recommended in recent guidelines to increase $\mathrm{VO}_{2 \text { peak }}$ in older people (Armstrong et al., 2006) . Therefore, in 2003, we started to study the effects of IWT, to repeat fast walking above $70 \%$ peak aerobic capacity for walking $\left(\mathrm{WV}_{2 \text { peak }}\right)$ for $3 \mathrm{~min}$ intermitted by slow walking below $40 \% \mathrm{WV}_{2 \text { peak }}$ at the target of 5 sets per day, more than 4 days/ 


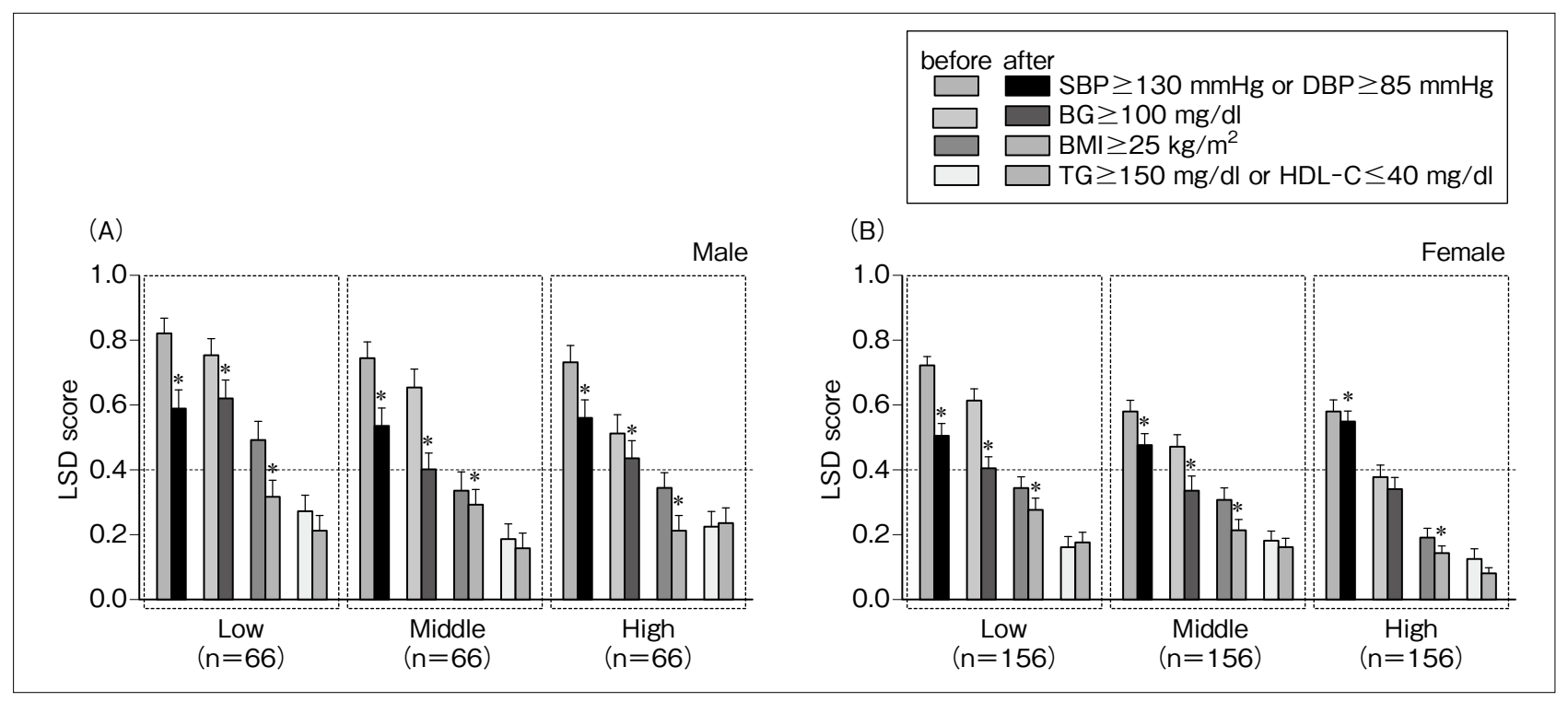

Figure-2 Lifestyle related disease (LSD) score for each criterion ; hypertension, hyperglycemia, high BMI, and dyslipidemia, in males (A) and females (B). When the subjects were divided equally into 3 groups according to $\mathrm{WVO}_{\text {2peak }}$ before training, the score was higher in the order of hypetension, hyperglycemia, high BMI, and dyslipidemia in every group. After training, all scores except for dyslipidemia decreased by $10-40 \%$. *'s : vs. before training at $\mathrm{p}<0.05$. From Morikawa et al., 2009.

week, for 5 months, on physical fitness for middleaged and older people (Nemoto et al., 2007). The reasons for adopting "interval" walking was that most subjects could not accomplish a training regimen by continuous fast walking $\geqq 15 \mathrm{~min} /$ day, $\geqq 4$ days/week, for 5 months in our preliminary study (unpublished data).

To determine $\mathrm{WV} \mathrm{O}_{2 \text { peak }}$, after baseline measurements at rest for 3 minutes, subjects with a triaxial accelerometer, as details below, on the midclavicular line of the waist walked on a flat floor at 3 graded subjective velocities:slow, moderate, and the fastest for 3min each while 3-dimensional accelerations with the device (JD Mate, Kissei Comtec, Matsumoto, Japan) and heart rate (HR) with a near infrared ear pickup probe were measured at 20-millisecond interval and recorded with 5-second memories as average value. The total impulse from an accelerometer was transferred to a computer and converted to oxygen consumption rate. $\mathrm{W} \dot{\mathrm{V}} \mathrm{O}_{2 \text { peak }}$ and peak $\mathrm{HR}$ are those for the last 30 seconds at the fastest velocity. We confirmed that the peak HR was $\sim 140$ beats/min, almost reaching the age expected maximal $\mathrm{HR}$ and that $\mathrm{WV} \mathrm{O}_{2 \text { peak }}$ $(\mathrm{ml} / \mathrm{min}, \mathrm{y})$ was quite identical to that determined by graded cycle ergometer exercise simultaneously determined on each subject. Thus, we can de- termine peak aerobic capacity on many subjects at once in the field with no limitation by instruments; treadmill and cycle ergometer.

Before the start of IWT, subjects were invited to a community office near their homes and received instruction in the exercise program for the first 2 weeks. Once subjects had learned the program, they could choose the time at which to perform it each day. A beeping signal from the device alerted subjects when a change of intensity was scheduled and another melody told them the time when walking intensity reached the target level every minute. Every 2 weeks subjects visited a local community office, and data from the tracking device were transferred to a central server at the administrative center through the Internet for automatic analysis by e-Health Promotion System and reporting. The details for this system are described below. Trainers used these reports to track daily walking intensity and other parameters given in to instruct subjects on how best to achieve the target levels. If the targets were not met, the trainers encouraged the subjects to increase their efforts to achieve them.

As a result, $\mathrm{WV} \mathrm{O}_{2 \text { peak }}$ increased by $\sim 10 \%$ and knee extension and flexion forces increased by $17 \%$ and $13 \%$, respectively, while systolic and dia- 
stolic pressures decreased by $\sim 10 \mathrm{mmHg}$ and $\sim 5$ $\mathrm{mmHg}$, respectively. On the other hand, their changes were all minimal after the standard walking training;moderate intensity continuous walking at $40 \% \mathrm{WV}_{2 \text { peak }}$ for $60 \mathrm{~min} /$ day, 4 days/week, for 5 months, which was similar to those after the sedentary life for the same period (Nemoto et al., 2007). Moreover, we found in the study that $\mathrm{W} \dot{\mathrm{V}} \mathrm{O}_{2 \text { peak }}$ was significantly correlated with isometric knee extension force $\left(\mathrm{R}^{2}=0.49, p<0.0001\right)$, suggesting that thigh muscle strength is a key determinant for $\mathrm{WV}_{2 \text { peak }}$ in subjects of this age. Further, these results indicate that increased $\mathrm{WV}_{2 \text { peak }}$ induced a marked reduction in blood pressures.

Although there were several epidemiological studies suggesting the merits of increased physical fitness to decrease the incidence of LSD including hypertension (Blair SN, et al., 1984), there have been few intervention studies by exercise training suggesting the linkage between them in a large population of middle-aged and older people. This might be because there have been no exercise training regimens to increase physical fitness broadly available for middle-aged and older people with less limitations of time and places.

Therefore, by using the techniques which we had developed, we examined the effects of IWT on physical fitness and the indices of LSD on 198 men and 468 women aged $\sim 65$ years old in 2005 and 2006 (Morikawa et al., 2009). They performed IWT, $\sim 60 \mathrm{~min} /$ day, $\sim 4$ days/week, for 4 months on average. We counted the scores of LSD before and after IWT according to the criteria in the health care guideline for Japanese by the government (Health Insurance Bureau, Ministry of Health, Labor, and Welfare, Japan 2007) ; 1) systolic blood pressure $\geqq 130 \mathrm{mmHg}$ or diastolic blood pressure $\geqq 85 \mathrm{mmHg}, 2$ ) triglyceride $\geqq 150 \mathrm{mg} / \mathrm{dl}$ or blood high density lipoprotein cholesterol $\leqq 40$ $\mathrm{mg} / \mathrm{dl}$, 3) blood glucose $\geqq 100 \mathrm{mg} / \mathrm{dl}$, 4) BMI $\geqq 25$ $\mathrm{kg} / \mathrm{m}^{2}$. Therefore, the full score was 4 points when met with all criteria.

To analyze the results, we divided the subjects into 3 groups according to $\mathrm{WV}_{2 \text { peak }}$ in females (Fig. 1-A) and males (Fig. 2-B). The LSD scores decreased as $\mathrm{WVO}_{2 \text { peak }}$ increased and, moreover, when $\mathrm{WV}_{2 \text { peak }}$ increased after training, the LSD score decreased in both genders. Further, when look at the LSD score in each criterion in females (Fig. 2-A) and males (Fig. 2-B), the hypertension score was $0.7-0.8$, suggesting that $70-80 \%$ of subjects met the criterion in both genders. Similarly, $40-60 \%$ and $20-50 \%$ of subjects were in hyperglycemia and high BMI, respectively, in both genders. After training, subjects met each criterion decreased by $5-30 \%$ in hypertension, $10-40 \%$ in hyperglycemia, and $10-30 \%$ in high BMI but with no significant reduction in blood lipids. These results suggest that increased $\mathrm{WV}_{2 \text { peak }}$ decreased blood pressures, blood glucose, and BMI in that order while the effects on blood lipids were modest.

To examine the effects of IWT on health care cost, we compared clinical expenditure between 166 participants ( 85 males and 81 females) in the program and counterbalanced 2,353 sedentary people (1,205 males and 1,148 females) aged $\sim 67$ years old who joined the National Health Insurance (unpublished data). Before IWT, the health care cost per person for the latter 6 months of 2004 was 87,649 JPY on average in the IWT group, similar to 87,746 JPY in the sedentary group, and for the first 6 months of 2005, it increased to 95,932 and 97,949 JPY, respectively, but with no significant difference between the groups. However, for the latter 6 months of 2005, the health care cost continued to increase to 119,173 JPY in the sedentary group while that in the IWT group remained unchanged as 96,272 JPY, 22,901JPY lower than the sedentary group with significance $(p<0.05)$. Thus, $23.8 \%$ of health care cost was saved by performing IWT.

\section{Portable calorie meter}

We have developed a new portable calorie meter with which energy expenditure can be precisely measured even when they walk on inclines (Yamazaki et al., 2009). First, we measured $\mathrm{W}_{\mathrm{V}} \mathrm{O}_{2}$ by respiratory gas analysis and vector magnitude (VM, G) from triaxial accelerations in middle-aged and older males and females aged $\sim 63$ years old during graded walking on a treadmill while the incline was varied from $-15 \%$ to $+15 \%$. They walked at subjectively slow, moderate and fast speeds on level and uphill inclines and in addition to these, at their fastest speed at $0 \%$ incline. Simi- 
larly, they then walked on downhill inclines for 3 min each. We determined a regression equation to estimate $\dot{\mathrm{V}} \mathrm{O}_{2}$ from $\mathrm{VM}$ and theoretical vertical up$\operatorname{ward} \operatorname{speed}(\mathrm{Hu}, \mathrm{m} / \mathrm{min})$ and downward speed $(\mathrm{Hd}$, $\mathrm{m} / \mathrm{min}$ ) for the last $1 \mathrm{~min}$ of each trail as $\dot{\mathrm{V}}_{2}=$ $0.0044 \mathrm{VM}+1.365 \mathrm{Hu}+0.553 \mathrm{Hd}$.

Second, to validate the precision of the equation, we measured VM and altitude changes with a portable device (JD Mate) equipped with a triaxial accelerometer and a barometer in middle-aged and older subjects walking on an outdoor hill, and compared the estimated $\dot{\mathrm{V}} \mathrm{O}_{2}$ by the equation stated above with the value simultaneously measured by respiratory gas analysis. We found that the estimated $\dot{\mathrm{V}}_{2}(\mathrm{y})$ from the equation was quite identical to the measured $\dot{\mathrm{V}} \mathrm{O}_{2}$ by respiratory gas analysis during walking on an outdoor hill. Thus, we have developed the device to estimate $\dot{\mathrm{V}} \mathrm{O}_{2}$ precisely during walking regardless of geography where subjects walk. Moreover, subjects can perform high-intensity exercise training $\geqq$ $70 \% \mathrm{WV}_{2 \text { peak }}$ not only by fast walking on a flat place but also by slow or moderate speed of walking on inclines or stairs.

\section{e-health promotion system}

Another reason for hindering us from extending exercise prescription for individuals to nationwide is the personnel cost for trainers who have the ability. To solve the problems, we have been developing the e-Health Promotion System (Nose et al., 2009) . The participants in the program visit local health care institutes near their homes; a local community office and a drug store, every 2 weeks, transfer their walking records from the JD Mate to a central sever computer, and receive a trend graph of their achievements. According to the records, the staff;nurses, dietitians, pharmacists or trainers, give them exercise and nutritional prescriptions while referring to the DB in the server computer about the effects of IWT for 5 months on physical fitness and the indices of LSD in 4,000 subjects and that for more than 5 month in 1,000 subjects. If participants have the facilities at their own homes, they can receive the same service through the Internet without going out.

\section{Individual genomic variance}

Recently, we have started to analyze individual genomic variance in relation with inter-individual variation in response to IWT (Mori et al, 2009, Masuki et al., 2010). Masuki et al. (2010) assessed whether single nucleotide polymorphism rs1042615 of the vasopressin V1a receptor altered the indices of LSD in the subjects and, if so, whether it also altered the effects of IWT. CC, CT, and TT carriers of rs1042615 (42, 118, and 64 men ; 113, 263, and 154 women, respectively) performed IWT, $\geqq 4$ days/wk, for 5 months. Before IWT, BMI and diastolic blood pressure for men were both higher in TT than in CC ; however the differences disappeared after IWT despite similar training achievement between groups. Moreover, after IWT, BMI and DBP decreased more in TT than in CC with a greater decrease in low-density lipoprotein (LDL) cholesterol in TT than CC. The decreases in DBP and LDL cholesterol were still greater in TT even after adjustment for their pretraining values. On the other hand, for women, these parameters before IWT and their changes after IWT were similar between CC, CT, and TT. Thus, polymorphism rs1042615 of the V1a receptor altered BMI and DBP in middle-aged and older men, and the training-induced responses of DBP and LDL cholesterol, whereas women did not show any of these responses. These results suggest that single nucleotide polymorphism rs1042615 of the vasopressin V1a receptor was involved in interindividual variance in responses to IWT in middle-aged and older men.

According to the outcome of these studies, we are developing a computer program to predict the effects of IWT on the physical fitness and the indices of LSD according to not only physical but also genetic characteristics of participants before training. If the program becomes available by the staff in the field, they would be able to give participants exercise and nutritional prescriptions more fitted for individuals even though they are not specialized in the subjects very much. This would increase the number of participants in IWT.

In conclusion, the IWT may significantly contribute the exercise prescription fitted for individual 
physical fitness broadly available in middle-aged and older people by using the JD Mate and the eHealth Promotion System. Moreover, IWT is such a simple intervention by exercise that it would enable us to develop exercise prescriptions more fitted for individuals including genetic characteristics.

\section{Acknowledements}

This study was supported in part by grants from the Ministry of Health, Labor, and Welfare (Comprehensive Research on Aging and Health), the Japan Society of Promotion of Science, and the Ministry of Economy, Trade, and industry of Japan. This research was also supported in part by the Shinshu University Partnership Project between Shinshu University, Jukunen Taiikudaigaku Research Center, the Ministry of Education, Culture, Sports, Science and Technology of Japan, and Matsumoto City.

\section{References}

1) Armstrong LE, Balady GJ, Berry MJ, et al : General Principles of exercise prescription. In : Whaley $\mathrm{MH}$, ed. ACSM's Guidelines for Exercise Testing and Prescription. $7^{\text {th }}$ ed. Baltimore, Md:Williams \& Wilkins, 2006 ; $133 \sim 173$.

2) Blair SN, Goodyear NN, Gibbons LW, et al : Physical fit- ness and incidence of hypertension in healthy normotensive men and women. JAMA, $1984 ; 252:$ 487 490 .

3) Health Insurance Bureau, Ministry of Health, Labor, and Welfare, Japan. Implement manual for health checkups and healthcare advice with a particular focus on the metabolic syndrome (in Japanese). 2007 ; 1 147.

4) Masuki S, Mori M, Tabara Y, for the Shinshu University Genetic Research Consortium. : Vasopressin V1a Receptor polymorphism and high-intensity interval walking training effects in middle-aged and older people. Hypertension, $2010 ; 55: 747 \sim 754$.

5) Mori M, Higuchi K, Sakurai A, et al : Genetic basis of inter-individual variance in the effects of exercise on preventing life-style related diseases. J Physiol, 2009 ; $587: 5577 \sim 5584$.

6) Morikawa M, Okazaki K, Masuki S, et al : Physical fitness and indices of lifestyle related diseases before and after interval walking training in middle-aged males and females. Br J Sports Med, in press.

7) Nemoto K, Gen-no H, Masuki S, et al : Effects of high-intensity interval walking training on physical fitness and blood pressure in middle-aged and older people. Mayo Clin Proc, 2009 ; 82 : 803 811.

8) Nose H, Morikawa M, Yamazaki T, et al : Beyond epidemiology : field studies and the physiology laboratory as the whole world. J Physiol, 2009 ; 587 : 5569 5575.

9) Yamazaki T, Gen-no H, Kamijo Y, et al : A new device to estimate VO2 during incline walking by accelerometry and barometry. Med Sci Sports Exerc, 2009 ; 41 : 2213 2219. 